\title{
Better Together?
}

The Cognitive Advantages of Synaesthesia for Time, Numbers and Space

\author{
Joanna Hale, Jacqueline M. Thompson \\ University of Oxford
}

Helen M. Morgan

Liverpool John Moores University

\author{
Marinella Cappelletti \\ University College London
}

Roi Cohen Kadosh

University of Oxford

Author Note

Joanna Hale, Jacqueline M. Thompson, Department of Experimental Psychology, University of Oxford; Helen M. Morgan, School of Natural Sciences and Psychology, Liverpool John Moores University; Marinella Cappelletti, Institute of Cognitive Neuroscience, University College London; Roi Cohen Kadosh, Department of Experimental Psychology, University of Oxford.

Joanna Hale is now at the Institute of Cognitive Neuroscience, University College London. Marinella Cappelletti is also at the Department of Psychology, Goldsmiths, University of London. 
The research was conducted at the University of Oxford. Roi Cohen Kadosh is supported by the Wellcome Trust, which funded this work under Grant WT88378. Marinella Cappelletti is sponsored by the Royal Society.

Correspondence should be addressed to Joanna Hale, UCL Institute of Cognitive Neuroscience, Alexandra House, 17 Queen Square, London, WC1N 3AR. Email jo.hale.13@ucl.ac.uk. Telephone 02076791126.

Jacqueline M. Thompson, Department of Experimental Psychology, Tinbergen Building, 9 South Parks Road, Oxford, OX1 3UD. Email jackie.thompson@psy.ox.ac.uk. Telephone 01865281245.

Helen M. Morgan, School of Natural Sciences and Psychology, Liverpool John Moores University, Tom Reilly Building, Byrom Street, Liverpool, L3 3AF. Email h.m.morgan@ljmu.ac.uk. Telephone 01519046336.

Marinella Cappelletti, UCL Institute of Cognitive Neuroscience, Alexandra House, 17 Queen Square, London, WC1N 3AR. Email m.cappelletti@ucl.ac.uk. Telephone 0207679 5430.

Roi Cohen Kadosh, Department of Experimental Psychology, Tinbergen Building, 9 South Parks Road, Oxford, OX1 3UD. Email roi.cohenkadosh@psy.ox.ac.uk. Telephone 01865271385. 


\begin{abstract}
Synaesthesia for time, numbers and space (TNS synaesthesia) is thought to have costs and benefits for recalling and manipulating time and number. There are two competing theories about how TNS synaesthesia affects cognition. The 'magnitude' account predicts TNS synaesthesia may affect cardinal magnitude judgements, whereas the 'sequence' account suggests it may affect ordinal sequence judgements and could rely on visuospatial working memory. We aimed to comprehensively assess the cognitive consequences of TNS synaesthesia and distinguish between these two accounts. TNS synaesthetes, graphemecolour synaesthetes and non-synaesthetes completed a behavioural task battery. Three tasks involved cardinal and ordinal comparisons of temporal, numerical and spatial stimuli; we also examined visuospatial working memory. TNS synaesthetes were significantly more accurate than non-synaesthetes in making ordinal judgements about space. This difference was explained by significantly higher visuospatial working memory accuracy. Our findings demonstrate an advantage of TNS synaesthesia which is more in line with the sequence account.
\end{abstract}

Keywords: synaesthesia, time, number, sequence-space, working memory 
Better Together?

The Cognitive Advantages of Synaesthesia for Time, Numbers and Space.

The recent renaissance of synaesthesia research has validated a diverse range of synaesthesias (Day, 2007; Deroy \& Spence, 2013; Simner, 2012) and provided an 'open window' for studying typical cognitive abilities (Cohen Kadosh \& Henik, 2007; Mulvenna \& Walsh, 2006; Sagiv \& Robertson, 2004). However, we do not have a clear understanding of how synaesthesia affects cognitive abilities, such as executive control (Mann, Korzenko, Carriere \& Dixon, 2009; Rouw, van Driel, Knip \& Ridderinkhof, 2013). As the estimated prevalence of synaesthesia has risen with more sophisticated research (Baron-Cohen, Burt, SmithLaittan, Harrison \& Bolton, 1996; Baron-Cohen et al., 2013; Sagiv, Simner, Collins, Butterworth \& Ward, 2006; Simner et al., 2006), it is timely to ask what cognitive abilities are affected by synaesthesia and what advantages it may have.

In this study, we examined a prevalent type of synaesthesia (Sagiv et al., 2006; Simner et al., 2006) which we call time, number and space (TNS) synaesthesia (Cohen Kadosh \& Gertner, 2011). In TNS synaesthesia, time and numbers are 'inducer' stimuli, which trigger the 'concurrent' experience of idiosyncratic spatial forms in mental or peripersonal space (Eagleman, 2009; Galton, 1880; Sagiv et al., 2006; Simner, 2009). Up to 24 per cent of the population may visualise time or number forms (Seron et al., 1992; Sagiv et al., 2006; see Brang, Teuscher, Ramachandran \& Coulson, 2010, for a more conservative estimate of time forms). Nevertheless, it is unclear whether TNS synaesthesia is a cognitive advantage when it comes to processing temporal, numerical or spatial information (Ward \& Sagiv, 2007).

\section{Costs and Benefits of Spatial Forms}

It is partly unclear whether TNS synaesthesia has cognitive advantages because existing studies show mixed effects. On the one hand, TNS synaesthesia seems to be an 
advantage for processing temporal information. TNS synaesthetes have enhanced abilities to remember meaningful dates (Simner, Mayo \& Spiller, 2009), count backwards in time (Mann et al., 2009) and learn new spatial forms for representing periods of time (Brang, et al., 2010). These advantages might be explained by TNS synaesthetes' enhanced performance on some tests of mental rotation and visual imagery (Simner et al., 2009), consistent with the hypothesis that spatial forms are a foundation for savant feats of recall (Murray, 2010; Rothen, Meier \& Ward, 2012; Simner et al., 2009). However, no studies have established whether mental rotation or visual imagery explains TNS synaesthetes' ability to recall and manipulate temporal information. The reported effects might alternatively stem from an auxiliary cognitive ability, such as visuospatial working memory.

On the other hand, spatial forms can impede cognitive flexibility when performing number and time tasks. Synaesthetes with number forms were significantly impeded in answering simple addition and multiplication problems (Ward et al., 2009), perhaps because they do not use rote strategies for addition and multiplication and instead employ visuospatial strategies which are less optimal for solving these problems (Zamarian, Ischebeck \& Delazer, 2009). Furthermore, synaesthetes make slower judgements about the order of months (Price \& Mentzoni, 2008; Smilek, Callejas, Dixon \& Merikle, 2007) and numbers (Gertner, Henik \& Cohen Kadosh, 2009; Hubbard, Ranzini, Piazza \& Dehaene, 2009) when these stimuli are incompatible with their spatial forms. These disadvantages could be due to accessing spatial forms automatically (Cohen Kadosh et al., 2012;

Diesendruck et al., 2010; Jarick, Dixon \& Smilek, 2011), although some have questioned the automaticity of TNS synaesthesia (Price \& Mattingley, 2013).

These studies of TNS synaesthesia suggest that experiencing spatial forms can have cognitive benefits in certain contexts and costs in others (Cohen Kadosh et al., 2012; Simner, 2009). However, it is not clear whether these costs and benefits are direct consequences of 
experiencing spatial forms. The results might have been due to TNS synaesthetes intentionally using their spatial forms in order to complete the tasks (Brang et al., 2010; Mann et al., 2009; Simner et al., 2009; Ward et al., 2009), in which case we would expect them to perform differently from non-synaesthetes even if both groups have similar cognitive ability.

We have identified two theoretical accounts of TNS synaesthesia which lead us to expect there are direct consequences of TNS synaesthesia on cognitive ability: a 'magnitude' account and a 'sequence' account. Both theories assume spatial forms result from heightened use of non-synaesthetic cognitive mechanisms, but they diverge over which cognitive mechanisms are involved (Sagiv et al., 2006). We discuss below the competing predictions inferred from these accounts and the different possibilities they imply for the role of visuospatial working memory in TNS synaesthesia. Following the debate between the magnitude and sequence accounts, our study sought to test whether TNS synaesthesia has cognitive advantages for magnitude judgements, sequence judgements or visuospatial working memory.

\section{The Magnitude Account}

The magnitude account suggests TNS synaesthesia builds on a non-synaesthetic mechanism for representing magnitudes (Cohen Kadosh \& Gertner, 2011; Gertner et al., 2012). This mechanism is described in A Theory of Magnitude (ATOM; Bueti \& Walsh, 2009; Walsh, 2003), which proposes that magnitudes of time, number, space and other dimensions are processed by one shared parietal mechanism (Bueti \& Walsh, 2009; Walsh, 2003). Via this mechanism, magnitudes such as numerical quantity are thought to be represented by a system that originally evolved for representing space (Bueti \& Walsh, 2009; Cohen Kadosh et al., 2008; Cohen Kadosh et al., 2012). The magnitude account argues TNS synaesthesia is an unusually strong tendency to consciously represent time, numbers and 
space as equivalent magnitudes, due to decreased specialisation of the parietal mechanism described in ATOM (Cohen Kadosh et al., 2012; Cohen Kadosh \& Gertner, 2011; Gertner, Arend \& Henik, 2012). The fact that synaesthetes often have three-dimensional spatial forms (Eagleman, 2009), whereas non-synaesthetes have shown behavioural effects indicating they spatially represent numbers and time in just one or two dimensions (Dehaene, Bossini \& Giraux, 1993; Boroditsky, 2000), may reflect the strength of the ATOM mechanism in synaesthesia.

Behavioural effects support the magnitude theory. The spatial-numerical association of response codes (SNARC) effect (Dehaene, Bossini \& Giraux, 1993; Fitousi, Shaki \& Algom, 2009) refers to the robust finding that people make faster left-hand responses about small numbers versus large numbers, and faster right-hand responses for the reverse (Dehaene et al., 1993; Wood, Willmes, Nuerk \& Fischer, 2008). This and similar 'SNARClike' effects for pitch (Rusconi, Kwan, Giordano, Umiltà \& Butterworth, 2006), weight (Holmes \& Lourenco, 2013) or even emotions (Holmes \& Lourenco, 2011), provide support for ATOM (Bueti \& Walsh, 2003). In support of the assumption that TNS synaesthesia is a conscious experience of the same phenomenon, there are unique SNARC effects which accompany spatial forms. Synaesthetes with vertical number forms experience a vertical SNARC effect (Jarick, Dixon, Maxwell, Nicholls \& Smilek, 2009), and synaesthetes with time forms for months demonstrate a special SNARC-like effect (Price \& Mentzoni, 2008, but see Gevers, Reynvoet \& Fias, 2003). Moreover, TNS synaesthetes seem to experience the classic SNARC effect especially strongly, supporting the idea that an unusually strong ATOM mechanism underlies TNS synaesthesia (Arend, Gertner \& Henik, 2013). Note, however, that another study was not able to replicate these findings (Jonas, Spiller, Jansari \& Ward, 2013). 
Importantly for the magnitude account, there are unique SNARC-like effects in TNS synaesthesia which cannot result from the ordinal relationships between abstract items like numbers or months. For example, TNS synaesthetes displayed a SNARC-like effect when comparing equal numbers of different physical size (Gertner, Henik, Reznik \& Cohen Kadosh, 2012) and experienced a SNARC-like effect for clusters of dots as well as Arabic numbers (Gertner, Arend \& Henik, 2012). This suggests the SNARC effects which accompany spatial forms result from the cardinal mapping of time and number magnitudes onto space, rather than the ordinal relationships between months and numbers (Gertner et al., 2012).

\section{The Sequence Account}

The sequence account directly opposes the magnitude account (Eagleman, 2009; Tang, Ward \& Butterworth, 2008). Its proponents argue TNS synaesthesia is a misnomer for sequence-space synaesthesia, and that the ordinal nature of inducers is the important feature of time and number forms (Eagleman, 2009; Murray, 2010; Rizza \& Price, 2009). The sequence account suggests over-learned sequences like integers, days of the week or months of the year are reified in synaesthesia to have the properties of visualised objects with spatial co-ordinates (thus relying on spatial co-ordinate systems in the parietal cortex; Eagleman, 2009; Sagiv et al., 2006).

The sequence account takes its strength from the nature of synaesthetes' spatial forms. Most compellingly, the inducers for spatial forms are not restricted to magnitudes like numbers and time (Eagleman, 2009). Many arbitrary sequences are spatially arranged by synaesthetes: the alphabet is a common inducer (Jonas, Taylor, Hutton, Weiss \& Ward, 2011), and more unusual ones include the Indian caste system and television schedules (Cytowic \& Eagleman, 2009; Simner, 2009). The existence of these forms, plus the fact that TNS synaesthetes seem to have enhanced mental rotation skills (Brang et al., 2013) and 
indeed may sometimes take different vantage points on their forms (Eagleman, 2009; Jarick, Dixon, Stewart, Maxwell \& Smilek., 2009), has been interpreted as sufficient evidence to accept the sequence account (Eagleman, 2009; Murray, 2010; Sagiv et al., 2006).

Neural evidence about TNS synaesthesia also favours the sequence account. An fMRI study found specific activity in the parietal lobes of synaesthetes when they performed an ordinal number task, but not a cardinal task with the same stimuli, despite this difference being undetectable at a behavioural level (Tang, Ward \& Butterworth, 2008). This neural effect suggests ordinal relationships among numbers are specially processed in TNS synaesthesia. Therefore, some synaesthetes may report spatial forms for continuous quantities like weights (Hubbard et al., 2009) the time of day (Simner et al., 2009), or temperature (reported by a synaesthete in the present study) because these inducers can be numerically sequenced (Simner, 2009). That is, even though evidence from SNARC-like effects suggests TNS synaesthesia involves the cardinal mapping of magnitudes onto space, it could actually involve processing magnitudes as if they are ordinal sequences instead.

\section{Visuospatial Working Memory}

The magnitude and sequence accounts imply different roles for visuospatial working memory in TNS synaesthesia. The magnitude account does not imply working memory is necessary in order to have a conscious experience of spatial forms. In contrast, the sequence account assumes that TNS synaesthesia is a process of visualisation in the co-ordinates of mental space (e.g. Eagleman, 2009; Price \& Mattingley, 2013), which is proposed to recruit the ventral visual stream for representing sequenced items as objects (Eagleman, 2009). In non-synaesthetes, this kind of visualisation is thought to happen in working memory (Fiore, Borella, Mammarella \& Cornoldi, 2011; Gyselinck, Beni, Pazzaglia, Meneghetti \& Mondoloni, 2007). Therefore, the sequence account raises the possibility that TNS synaesthetes maintain their spatial forms in visuospatial working memory, although this 
possibility has not been previously discussed. Since there is very little existing theory or evidence about the role of visuospatial working memory in TNS synaesthesia, it will be important to test whether visuospatial working memory ability can account for the effects of spatial forms on the ability to process time, numbers or space. One study found that spatial working memory is not altered in TNS synaesthesia (Brang, Miller, McQuire, Ramachandran \& Coulson, 2013), but only measured spatial working memory span ranging from one to five items, which may lack sensitivity. Visuospatial working memory has both visual and spatial components (Kozhevnikhov, Kosslyn \& Shephard, 2005; Mohr \& Linden, 2005), associated with ventral and dorsal visual streams, respectively (Jackson, Morgan, Shapiro, Mohr \& Linden, 2011). The sequence account particularly highlights the importance of ventral visual processing in TNS synaesthesia. Therefore, separately measuring visual and spatial components of working memory with a sensitive task could give us new insight into the role of working memory in TNS synaesthesia.

In this study we aimed to assess the cognitive advantages of TNS synaesthesia, directly compare the magnitude and sequence accounts and examine the role of working memory in TNS synaesthesia. To fulfil this aim we designed a task battery including three tasks which required participants to make comparative judgements about time, number and space stimuli. Each task was manipulated to require either ordinal or cardinal judgements about identical stimuli (c.f. Cheng et al., 2012; Tang et al., 2008), so that we could compare the predictions made by the magnitude and sequence accounts. Unlike previous tasks without a time limit (Brang et al., 2010; Mann et al., 2009; Simner et al., 2009), these three tasks were designed to measure relatively fast response times, so that spatial forms could not be used voluntarily. However, in case TNS synaesthesia only has advantages when used voluntarily, we also included a task in which spatial forms could be exploited to compare identical digits as numbers and as times (clock-times or dates). To examine the role of visuospatial working 
memory, we administered a task which measures visual and spatial components separately. We applied an EZ-diffusion model (Wagenmakers et al., 2007) to the reaction time and accuracy data from each task, to derive a measure of overall task ability (i.e., drift rate). ). Aside from recruiting a relatively large numbers of synaesthetes in this study, we also controlled for any motivational effects of synaesthesia by comparing TNS synaesthetes to grapheme-colour (GC) synaesthetes as well as non-synaesthetes controls (c.f. Gheri, Chopping \& Morgan, 2008). GC synaesthesia provides a good control for TNS synaesthesia because it has similar prevalence (Eagleman et al., 2007; Sagiv et al., 2006) and also links visual inducers (which may be numbers) with a visual concurrent.

\section{Methods}

\section{Participants}

Twenty-three TNS synaesthetes ${ }^{1}$ ( 6 male; age $M=24.1, S D=4.6$ ), $21 \mathrm{GC}$ synaesthetes ( 4 male; age $M=25.6, S D=7.6$ ) and 25 non-synaesthetes (12 male; age $M=$ $24.4, S D=7.9)$ provided written consent to participate in the study, which was approved by the Central University Research Ethics Committee, University of Oxford. The gender ratios of the three groups did not significantly differ $\left(\chi^{2}(2, N=69)=4.94, p=.09\right)$. However, we tested whether gender affected our results by including gender as a covariate in the analyses reported in the Results section.

All synaesthetes completed a semi-structured synaesthesia interview with open questions asking participants to describe all inducers and concurrents they experience plus idiosyncratic characteristics of their experiences, like the shape of spatial forms or specific grapheme-colour parings. The types of spatial forms our participants reported are summarised in Table 1. This interview was used to assign people to the synaesthesia conditions for two main reasons: First, no suitable tests have been sufficiently developed to verify TNS synaesthesia (c.f. Eagleman, 2009). Second, Simner (2012) has suggested recently that the 
current use of test-retest, which is used to check consistency in GC synaesthesia (e.g. Eagleman, Kagan, Nelson, Sagaram \& Sarma, 2007), might exclude potential synaesthetes whose synaesthetic concurrents are not sufficiently consistent to pass the test-retest requirement. This could be a risk in TNS synaesthesia, as Brang et al. (2010) reported that only four out of 81 'potential synaesthetes' had significantly higher consistency than controls. Therefore we decided not to use the test-retest method to define people as synaesthetes. Instead we expected to find group differences, in line with our predictions, which would corroborate the interviews and also allow a more representative sample of the syanesthete population (see Simner, 2012). Our results support the validity of our approach. Only TNS synaesthetes reporting no grapheme-colour correspondences and GC synaesthetes reporting no spatial forms were invited to participate. To ensure non-synaesthete participants did not experience spatial forms or grapheme-colour correspondences, they were asked two questions prior to participation: (1) Do you see time or numbers in space? (2) Do you strongly associate certain colours with letters or numbers? (Gertner et al., 2009).

(Table 1 about here)

\section{Procedure}

Each participant completed a battery of five tasks, in the following order: Time, Number, Space, Clock-Date and Working Memory. This order was used so that all participants could have a break at a consistent time during the battery. The Time and Number tasks took approximately one hour to complete; each participant then took a break of at least five minutes, and up to 15 minutes, before completing the remaining tasks in around 45 minutes.

The Time task stimuli were computer-administered, but participants received spoken instructions and were asked to respond orally to the experimenter, under no time limit. Their accuracy was recorded. The remaining tasks were fully computer-administered and 
participants were asked to respond with a key-press as quickly and accurately as possible, within a limit of three seconds. The key-press responses were left $(Z)$ for 'yes' and right (M) for 'no', or vice versa (counterbalanced between-participants, within-groups), using a QWERTY keyboard.

\section{Experimental tasks}

Time, number and space. The first three tasks in the battery measured time, number or space judgements. Each task included a magnitude and a sequence manipulation, which were counterbalanced between-participants, within-groups.

Time. Ability to make comparative judgements about time was measured with a task adapted from Cappelletti, Freeman \& Cipolotti (2009). Participants were presented with a sequence of coloured circles which appeared one at a time in the centre of the screen (see Figure 1a and Appendix for stimulus details), and were asked to name each colour aloud, to prevent the use of sub-vocal strategies for the task. In the magnitude manipulation, participants were instructed to estimate the duration of the sequence in seconds. To measure accuracy, the absolute difference between their estimation and the veridical trial duration was computed as a percentage of the trial duration. In the sequence manipulation, a target (' $\mathrm{T}$ ') appeared during the sequence, and participants were instructed to estimate whether more circles appeared before or after the target ${ }^{2}$. To measure accuracy, responses were simply coded as correct or incorrect. Each manipulation had 24 trials.

Number. Ability to make comparative judgements about number was measured with the task used by Tang et al. (2008). Participants were presented with a string of three to five characters, of which one was an Arabic numeral, 1-7, and the others were the letter X (see Figure $1 \mathrm{~b}$ and Appendix for stimulus details). In the magnitude manipulation, participants were instructed to judge whether the numeral was equal to the number of characters. In the sequence manipulation, participants were instructed to judge whether the numeral was in the 
position of that number in the string of characters, counting from the left or the right (counterbalanced within-participants). Each manipulation had 216 trials.

Clock-Date. This task measured the ability to compare between stimuli in the two domains which provide inducers in TNS synaesthesia: time and number. The task exploits the fact that numerals can be used to denote numbers or time, as in a digital clock time or a date (c.f. Cappelletti, Lee, Freeman \& Price, 2009; Cappelletti, Muggleton \& Walsh, 2009). Participants were presented with a pair of two-digit numbers, shown above and below a fixation cross to avoid bias in left and right key-press responses (see Figure 1d and Appendix for stimulus details). There were two manipulations of the task, each with 48 trials: In the clock manipulation, participants were instructed to judge whether the larger number was also the later time in minutes past an hour if the numbers were read as times on a digital clock. In the date manipulation, participants were instructed to judge whether the larger number was also the later date by position in the year if the numbers were read as dates in DD.MM format.

Space. We designed a novel task to measure ability to make comparative judgements about spatial stimuli. Participants were presented with a set of three overlapping circles, one large, one medium and one small (see Figure 1c and Appendix for stimulus details). In the magnitude manipulation, participants were instructed to judge whether the topmost overlapping circle was the largest or the smallest (counterbalanced within-participants). In the sequence manipulation, the size of the circles was irrelevant; participants were instructed to judge whether the circles were overlapping in order with the leftmost on the bottom and the rightmost on the top, or vice versa (counterbalanced within-participants). Each manipulation had 72 trials.

Visuospatial Working Memory. Visual, spatial and combined visuospatial working memory were measured in the final task (Morgan, Muthukumaraswamy, Singh \& Linden, 
2013). Participants were presented with a sample of 1 to 4 (low-high working memory load) coloured semicircles around a central fixation point; then, after a two-second delay, a single coloured semicircle was presented (see Figure 2). There were three manipulations of the task, each with 24 trials: In the angle matching manipulation, participants were instructed to judge whether the singly presented semicircle matched any of the preceding sample in its exact angle of rotation. In the colour matching manipulation, participants were instructed to judge whether it matched in exact hue. In the dual matching manipulation, participants were instructed to judge whether it matched in exact angle of rotation and hue. The first two manipulations measure separable visual and spatial components of visuospatial working memory (Jackson et al., 2011; Mohr \& Linden, 2005), whilst the dual manipulation should measure combined visuospatial working memory with more validity than averaging ability across angle and colour matching (Jackson et al., 2011).

\section{Data Analysis}

Missing data. One non-synaesthete's data was missing from the Number task, due to misunderstanding the instructions. Two TNS synaesthetes' data was missing data from the Space task, due to random failure of the task programme. One GC synaesthete's data was missing from the Clock-Date task, and another's from the Working Memory task, due to running out of time. Participants with missing data were excluded case-wise from the main analyses, since the sample was large and the reasons for missing data were unlikely to be related to synaesthesia or task manipulations, which were the factors tested for an effect (cf., Graham, Cumsille \& Elek-Fisk, 2003; Schafer \& Graham, 2002).

Main analyses. A series of two-way mixed-design ANOVAs were conducted. Each ANOVA tested the between-participants factor of synaesthesia (TNS synaesthesia, GC synaesthesia or non-synaesthesia) and the within-participants factor of task manipulation (magnitude or sequence; clock or date; angle, colour or dual matching), and their interaction. 
For the Time task, one ANOVA tested the effects of the factors on accuracy. For the Number, Space and Clock-Date tasks, separate ANOVAs tested the effects of the factors on accuracy and response time for correct answers.

Any ANOVAs resulting in a significant effect of synaesthesia in time, number or space were repeated as ANCOVAs, controlling for Working Memory performance. This was to test the prediction, following the sequence account, that controlling for visuospatial working memory may have a greater effect on group differences in magnitude judgement than sequence judgement. A three-way mixed design ANOVA testing the betweenparticipants factor of synaesthesia (TNS synaesthesia, GC synaesthesia or non-synaesthesia) and the within-participants factors of subtask (angle, colour or dual matching) and load (1, 2, 3 or 4 items) was also conducted to explore visuospatial working memory ability across each group.

EZ-diffusion modelling. In order to interpret the ability of our participants from accuracy and response time data, we applied an EZ-diffusion model (Wagenmakers, van der Maas \& Grasman, 2007). The EZ-diffusion model shares a conceptual basis with full diffusion modelling, but computes just three parameters which are most informative about an individual's task performance: task ability (or 'drift rate'), which is the variable of interest for the present study; response conservativeness (or 'boundary separation'), i.e., the threshold for making a response; and non-decision time, i.e., the time taken to perceive a stimulus before executing a decision about it. The equations for calculating task ability and the other parameters in the model can be derived entirely from the proportion of correct trials, mean response time for correct trials and variance in response time for correct trials. The mathematical derivation is explained in detail in Wagenmakers et al. (2007). The task ability of a given participant is calculated as a function of the proportion of trials where they gave a correct response, divided by their variance in response time for correct responses. 
Response time data should meet three basic assumptions to apply the EZ-diffusion model (Wagenmakers, van der Maas, Dolan \& Grasman, 2008). It is assumed that each participant's response times are right-skewed, do not differ between correct and incorrect responses, and do not show a response bias among incorrect responses. The first assumption was met for three of our binary forced-choice tasks (Number, Space and Working Memory). The second and third assumptions could not be tested for each participant individually, because they made too few errors on these tasks. However, we found no difference between correct and incorrect reaction times and no response bias in the distribution of average reaction times for the whole sample. Therefore, we applied the EZ-diffusion model to our data. Further tests confirmed TNS synaesthetes, GC synaesthetes, and non-synaesthetes did not differ in response conservativeness or non-decision time, meaning any reported differences in task ability were not due to these components of task performance. Therefore, we examined any significant effects found in the main analyses by conducting the same test on the task ability scores derived from the EZ-diffusion model.

\section{Results}

The main analyses revealed several significant advantages of TNS synaesthesia (see Figure 3). We only report the main analyses for the Space and Working Memory tasks as no significant effects of synaesthesia were found in the other tasks (all $F \mathrm{~s}<1.4$, all $p \mathrm{~s}>.05$ ). Further analyses examined the effects in these two tasks and whether they were linked.

\section{Space Task Accuracy}

For the Space task, an $\mathrm{ANOVA}^{3}$ testing the between-participants factor of synaesthesia and the within-participants factor of manipulation (magnitude or sequence) found a significant main effect of synaesthesia $\left(F(2,61)=3.75, p=.03, \eta_{\mathrm{p}}{ }^{2}=.11\right)$ and a significant main effect of manipulation $\left(F(2,61)=24.32, p<.001, \eta_{\mathrm{p}}^{2}=.29\right)$ on accuracy. There was also a significant interaction between synaesthesia and manipulation $(F(2,61)=$ 
$\left.3.36, p=.04, \eta_{\mathrm{p}}{ }^{2}=.09\right)$. The same interaction approached significance in the task ability data derived from the EZ-diffusion model $\left(F(2,64)=3.00, p=.06, \eta_{\mathrm{p}}^{2}=.08\right)$.

Pair-wise comparisons revealed that the interaction reflected two effects. First, TNS synaesthetes had significantly higher accuracy $(M=.95,95 \%$ CI $[.89,1.00])$ than nonsynaesthetes $(M=.84,95 \% \mathrm{CI}[.79, .90])$ in the sequence manipulation $(t(43)=2.78, p=$ .008 , Cohen's $d=0.94)$, but not in the magnitude manipulation $(t(43)=1.22, p=.23$, Cohen's $d=0.33$ ). In the task ability data derived from the EZ-diffusion model, the difference between TNS synaesthetes and non-synaesthetes was also significant in the sequence manipulation $(t(44)=3.21, p=.002$, Cohen's $d=0.97)$. Second, whilst nonsynaesthetes and GC synaesthetes were significantly better at processing magnitude than sequence information, TNS synaesthetes did not differ significantly across the manipulations of the Space task (see Figure 3).

To see that this interaction effect reflected a true difference between the magnitude and sequence subtask and not ceiling effects in the magnitude task, we divided participants' performance on the magnitude manipulation by quartiles and included magnitude performance as a 4-level factor in the ANOVA. This additional factor had a main effect on Space task performance $\left(F(3,64)=4.57, p=.007, \eta_{\mathrm{p}}{ }^{2}=.21\right)$; however, magnitude performance did not have any significant interactions with the other factors (see Figure 4): the effect of group was comparable across all four levels of magnitude performance (group X quartile: $\left.F(6,64)=.32, p=.92, \eta_{\mathrm{p}}^{2}=.04\right)$, as was the effect of task manipulation (task $\mathrm{X}$ quartile: $\left.F(3,64)=.75, p=.53, \eta_{\mathrm{p}}{ }^{2}=.04\right)$ and the interaction between group and task manipulation (group X task X quartile: $F(6,64)=.45, p=.84, \eta_{\mathrm{p}}^{2}=.05$ ).

Because significant effects of synaesthesia and task manipulation were found, the same ANOVA was carried out with Working Memory accuracy as a covariate. Holding Working Memory accuracy constant attenuated the significant main effect of synaesthesia on 
Space task accuracy $\left(F(2,61)=2.22, p=.12, \eta_{\mathrm{p}}^{2}=.07\right)$, as well as the interaction between synaesthesia and manipulation $\left(F(2,61)=1.95, p=.15, \eta_{\mathrm{p}}^{2}=.06\right)$. Notably, the covariate Working Memory accuracy was significantly correlated with Space task accuracy $(F(1,61)=$ $\left.6.18, p=.02, \eta_{\mathrm{p}}^{2}=.10\right)$. As Working Memory accuracy is an average of accuracy on each task manipulation, angle, colour or dual matching, these results do not reveal which component of visuospatial working memory may explain TNS synaesthetes' advantage in processing ordinal spatial information. Therefore, the same model was also tested with accuracy from each task manipulation (angle, colour or dual matching) as a covariate, to allow better resolution regarding these specific working memory components. Controlling for angle or colour matching accuracy significantly diminished the effect of synaesthesia to similar extents, whilst controlling for dual matching accuracy did not attenuate the main effect of synaesthesia (Table 2).

(Table 2 about here)

\section{Working Memory Task}

For the Working Memory task, an ANOVA testing the between-participants factor of synaesthesia and the within-participants factors of manipulation (angle, colour or dual matching) and load (1, 2, 3 or 4 items) found only a significant main effect of load $(F(3,64)$ $\left.=100.30, p<.001, \eta_{\mathrm{p}}^{2}=.61\right)$ on accuracy. However, since the combined visuospatial working memory in the dual task manipulation is suggested to reflect more executive demands (Mohr \& Linden, 2005), and because only angle matching and colour matching had a mediating role in the effects of synaesthesia on Space task accuracy, the same ANOVA was conducted with the dual matching task manipulation excluded. This ANOVA revealed a significant main effect of synaesthesia on Working Memory task accuracy $(F(2,64)=3.44$, $\left.p=.04, \eta_{\mathrm{p}}{ }^{2}=.10\right)$ as well as the main effect of $\operatorname{load}\left(F(3,64)=61.1, p<.001, \eta_{\mathrm{p}}{ }^{2}=.49\right)($ see Figure 5). Examining the main effect of synaesthesia more closely, pair-wise comparisons 
showed that TNS synaesthetes had significantly higher accuracy $(M=.83,95 \%$ CI $[.80, .86])$ than non-synaesthetes $(M=.78,95 \% \mathrm{CI}[.76, .81], p=.02)$. GC synaesthetes' accuracy $(M=$ $.81,95 \%$ CI $[.78, .84])$ did not significantly differ from either non-synaesthetes' $(p=.59)$ or TNS synaesthetes' $(p=.75)$.

\section{Discussion}

We found that TNS synaesthetes had an advantage in spatial processing ability. This cognitive advantage was specific to the domain of their synaesthetic concurrent (space), and not inducers (time or number). Specifically, TNS synaesthetes were significantly more accurate than non-synaesthetes in responding to spatial stimuli when their responses required an ordinal judgement. This cognitive benefit could be explained by either visual or spatial working memory, as TNS synaesthetes had elevated accuracy in both these components of visuospatial working memory, and controlling for either component attenuated their significant advantage in making ordinal spatial judgements. Where previous studies found significant effects of synaesthesia on recall (Simner et al., 2009), mental rotation (Simner et al., 2009; Brang et al., 2010; Brang et al., 2013) and mental calculations (Ward et al., 2009), our study suggests those effects are likely due to an advantage in spatial processing coupled with highly accurate visuospatial working memory.

In our tasks, TNS synaesthetes and non-synaesthetes did not differ in their ability to make comparative judgements about time and number stimuli. We replicated Tang et al.'s (2008) finding that TNS synaesthetes do not have an advantage in either cardinal or ordinal number processing. While Tang et al. (2008) found activity in the IPS unique to TNS synaesthetes' ordinal number processing our replication of their null findings at a behavioural level suggests this neural effect is not linked with ordinal judgements of number at the 
cognitive level. Rather, enhanced spatial processing in TNS synaesthesia could account for the IPS activity found by Tang et al. (2008).

The specific advantage we observed in the Space task suggests this effect was not driven by motivation, otherwise an advantage would have been observed in more than one task. Furthermore, to control for the fact that TNS synaesthetes were specially recruited, are interested in psychological research about their experiences (Ward, 2008) and therefore might have made more effort (Gheri et al., 2008; Simner et al., 2009), GC synaesthetes were also recruited using the same procedures. Since GC synaesthetes were not significantly different from controls in any way, the differences between TNS synaesthetes and controls should reflect a true effect of TNS synaesthesia.

\section{The Magnitude vs. Sequence Debate}

The significant advantages we found in the Space and Working Memory tasks are consistent with a sequence account of TNS synaesthesia. The effect of TNS synaesthesia in the Space task was limited to the sequence manipulation, in which participants had to judge whether a sequence of three overlapping circles was layered in a specified order (leftwards or rightwards). When participants had to judge whether the topmost circle was of the specified size (largest or smallest), group differences did not reach significance. As the stimuli were identical across manipulations, we can assume the manipulations differed only in the type of cognitive process required to make a response (Cheng et al., 2012; Tang et al., 2008). Therefore, this result fits the sequence account prediction that TNS synaesthetes have an ordinal rather than cardinal advantage when making spatial comparisons (Eagleman, 2009).

TNS synaesthetes and non-synaesthetes did not differ in their ability to make ordinal judgements about space when visuospatial working memory was held constant. This falls in line with our interpretation of the sequence account, because it suggests that the advantage TNS synaesthetes have for ordinal spatial processing can be explained by visuospatial 
working memory. Our results are not entirely consistent with the assumption that TNS synaesthesia primarily relies on visualising the items of a sequence as objects, because controlling spatial working memory led to an equal reduction in the effect of synaesthesia as controlling visual, object-like working memory. However, it could be that spatial encoding of the stimuli in our working memory task aided retention of their visual properties, and vice versa. As well as accounting for the relationship between visual working memory and spatial processing, this would explain why TNS and GC synaesthetes had similar performance despite experiencing spatial versus visual concurrents, respectively. Since the magnitude account assumes spatial forms do not rely on working memory, the findings are more consistent with the sequence account.

It is worth noting that GC synaesthetes had a profile of intermediate performance which is not easily explained by a 'magnitude' or 'sequence' framework. Their accuracy at judging spatial sequences was about halfway between TNS and non-synaesthetes, high enough that they did not significantly differ from TNS synaesthetes. As a speculative explanation for this pattern, GC synaesthetes may experience spatial working memory advantages beyond the visual domain of their concurrent (colour; Terhune, Wudarczyk, Kochuparampil and Cohen Kadosh, 2013), giving them elevated accuracy in the Space task. This could also explain the high co-occurence of TNS and GC synaesthesia (Sagiv et al., 2006), although our study specifically recruited participants with either TNS or GC synaesthesia.

\section{Visuospatial Working Memory}

Our TNS synaesthetes performed better than non-synaesthetes in visual and spatial working memory tasks. This is not only consistent with our interpretation of the sequence account, but it also highlights the possibility that cognitive benefits of TNS synaesthesia found in previous studies could be attributed to visuospatial working memory abilities. For 
example, unusually accurate maintenance of stimuli in visuospatial working memory might explain why TNS synaesthetes have shown advantages in recalling and mentally rotating manipulating stimuli which are inducers to their spatial forms (Brang et al., 2010, Mann et al., 2009; Simner et al., 2009), yet appear to automatically access their spatial forms to a disadvantage on certain mathematical tasks (Gertner et al., 2009; Hubbard et al., 2009; Ward et al., 2009).

\section{The Potential Effect of Gender}

We examined the potential effect of gender on our observed results by including it as a covariate. This addition did not affect the significant results we reported (all $p s<.05$ ).

\section{Conclusions}

This study was the first to comprehensively examine how ordinal and cardinal processing of time, numbers and space are affected by TNS synaesthesia and what cognitive advantages TNS synaesthesia may have. We found that TNS synaesthetes have a cognitive advantage for making ordinal judgements about space (their synaesthetic concurrent), which was explained by elevated visuospatial working memory ability. This result was consistent with our interpretation of the sequence account. It suggests that cognitive advantages of TNS synaesthesia may not come from using spatial forms, but from an underlying difference in working memory between TNS synaesthetes and non-synaesthetes. 


\section{References}

Arend, I., Gertner, L., \& Henik, A. (2013). Perceiving numbers influences actions in numberspace synesthesia. Cortex, 49(7), 1955-1962. doi:10.1016/j.cortex.2012.04.019

Baron-Cohen, S., Burt, L., Smith-Laittan, F., Harrison, J., \& Bolton, P. (1996). Synaesthesia: prevalence and familiality. Perception, 25(9), 1073-1079.

Baron-Cohen, S., Johnson, D., Asher, J., Wheelwright, S., Fisher, S. E., Gregersen, P. K., \& Allison, C. (2013). Is synaesthesia more common in autism? Molecular Autism, 4(1), 40. doi:10.1186/2040-2392-4-40

Boroditsky, L. (2000). Metaphoric structuring: Understanding time through spatial metaphors. Cognition, 75(1), 1-28. doi:10.1016/S0010-0277(99)00073-6

Brang, D., Miller, L. E., McQuire, M., Ramachandran, V. S., \& Coulson, S. (2013). Enhanced mental rotation ability in time-space synesthesia. Cognitive Processing. doi:10.1007/s10339013-0561-5

Brang, D., \& Ramachandran, V. S. (2011). Survival of the synesthesia gene: Why do people hear colors and taste words? PLoS Biology, 9(11). e1001205. doi:10.1371/journal.pbio.1001205

Brang, D., Teuscher, U., Miller, L. E., Ramachandran, V. S., \& Coulson, S. (2011). Handedness and calendar orientations in time-space synaesthesia. Journal of Neuropsychology, 5(2), 323332. doi:10.1111/j.1748-6653.2011.02012.x

Brang, D., Teuscher, U., Ramachandran, V. S., \& Coulson, S. (2010). Temporal sequences, synesthetic mappings, and cultural biases: The geography of time. Consciousness and Cognition, 19(1), 311-320. doi:10.1016/j.concog.2010.01.003

Bueti, D., \& Walsh, V. (2009). The parietal cortex and the representation of time, space, number and other magnitudes. Philosophical Transactions of the Royal Society B: Biological Sciences, 364(1525), 1831-1840. doi:10.1098/rstb.2009.0028 
Cappelletti, M., Freeman, E. D., \& Cipolotti, L. (2011). Numbers and time doubly dissociate. Neuropsychologia, 49(11), 3078-3092. doi:10.1016/j.neuropsychologia.2011.07.014

Cappelletti, M., Lee, H. L., Freeman, E. D., \& Price, C. J. (2009). The role of right and left parietal lobes in the conceptual processing of numbers. Journal of Cognitive Neuroscience, 22(2), 331-346. doi:10.1162/jocn.2009.21246

Cappelletti, M., Muggleton, N., \& Walsh, V. (2009). Quantity without numbers and numbers without quantity in the parietal cortex. NeuroImage, 46(2), 522-529. doi:10.1016/j.neuroimage.2009.02.016

Cheng, G. L. F., Tang, J., Walsh, V., Butterworth, B., \& Cappelletti, M. (2012). Differential effects of left parietal theta-burst stimulation on order and quantity processing. Brain Stimulation, 6(2), 160-165. doi:10.1016/j.brs.2012.04.005

Cohen Kadosh, R., \& Gertner, L. (2011). Synaesthesia: Gluing together time, number and space. In S. Dehaene \& E. M. Brannon (Eds.), Space, Time and Number in the Brain, Attention \& Performance (pp. 123-132). Elsevier.

Cohen Kadosh, R., Gertner, L., \& Terhune, D. (2012). Exceptional abilities in the spatial representation of numbers and time: Insights from synesthesia. The Neuroscientist, 18, 20815.

Cohen Kadosh, R., \& Henik, A. (2007). Can synaesthesia research inform cognitive science?. Trends in Cognitive Sciences, 11(4), 177-184.

Cohen Kadosh, R., Henik, A., Rubinsten, O., Mohr, H., Dori, H., van de Ven, V., ... Linden, D. E. J. (2005). Are numbers special? Neuropsychologia, 43(9), 1238-1248. doi:10.1016/j.neuropsychologia.2004.12.017

Cohen Kadosh, R., Tzelgov, J., \& Henik, A. (2008). A synesthetic walk on the mental number line: The size effect. Cognition, 106, 548-57. 
Cohen Kadosh, R., \& Walsh, V. (2009). Numerical representation in the parietal lobes: Abstract or not abstract? Behavioral and Brain Sciences, 32(3-4), 313. doi:10.1017/S0140525X09990938

Cytowic, R. E., \& Eagleman, D. M. (2009). Wednesday is indigo blue: Discovering the brain of synesthesia. Cambridge, MA: MIT Press.

Day, S. (2005). Some demographic and socio-cultural aspects of synesthesia. In L. Robertson, N. Sagiv (Eds.), Synesthesia: Perspectives from cognitive neuroscience (pp 11-33). New York: Oxford University Press.

Dehaene, S., Bossini, S., \& Giraux, P. (1993). The mental representation of parity and number magnitude. Journal of Experimental Psychology: General, 122(3), 371-396. doi:10.1037/0096-3445.122.3.371

Deroy, O., \& Spence, C. (2013). Why we are not all synesthetes (not even weakly so). Psychonomic Bulletin \& Review, 20(4), 643-664. doi:10.3758/s13423-013-0387-2

Diesendruck, L., Gertner, L., Botzer, L., Goldfarb, L., Karniel, A., \& Henik, A. (2010). Months in space: Synaesthesia modulates attention and action. Cognitive Neuropsychology, 27(8), 665679. doi:10.1080/02643294.2011.599796

Dixon, M. J., Smilek, D., \& Merikle, P. M. (2004). Not all synaesthetes are created equal: Projector versus associator synaesthetes. Cognitive, Affective, \& Behavioral Neuroscience, 4(3), 335-343. doi:10.3758/CABN.4.3.335

Eagleman, D. M. (2009). The objectification of overlearned sequences: A new view of spatial sequence synesthesia. Cortex, 45(10), 1266-1277. doi:10.1016/j.cortex.2009.06.012

Eagleman, D. M., Kagan, A. D., Nelson, S. S., Sagaram, D., \& Sarma, A. K. (2007). A standardized test battery for the study of synesthesia. Journal of Neuroscience Methods, 159(1), 139-145. doi:10.1016/j.jneumeth.2006.07.012 
Fiore, F., Borella, E., Mammarella, I. C., \& Cornoldi, C. (2011). Mental imagery in a visuospatial working memory task and modulation of activation. Journal of Cognitive Psychology, 23(1), 52-59. doi:10.1080/20445911.2011.454497

Fitousi, D., Shaki, S., \& Algom, D. (2009). The role of parity, physical size, and magnitude in numerical cognition: The SNARC effect revisited. Perception \& Psychophysics, 71(1), 143155. doi:10.3758/APP.71.1.143

Galton, F. (1880). Visualised Numerals. Nature, 21, 323. doi:10.1038/021323a0

Gertner, L., Arend, I., \& Henik, A. (2012). Effects of non-symbolic numerical information suggest the existence of magnitude-space synesthesia. Cognitive processing, 13( 1), 179-183. doi:10.1007/s10339-012-0449-9

Gertner, L., Henik, A., \& Cohen Kadosh, R. (2009). When 9 is not on the right: Implications from number-form synesthesia. Conscious Cognition, 18, 366-74.

Gertner, L., Henik, A., Reznik, D., \& Cohen Kadosh, R. (2012). Implications of number-space synesthesia on the automaticity of numerical processing. Cortex. doi:10.1016/j.cortex.2012.03.019

Gevers, W., Reynvoet, B., \& Fias, W. (2003). The mental representation of ordinal sequences is spatially organized. Cognition, 87(3), 87-95.

Gheri, C., Chopping, S., \& Morgan, M. J. (2008). Synaesthetic colours do not camouflage form in visual search. Proceedings of the Royal Society B: Biological Sciences, 275(1636), 841-846. doi:10.1098/rspb.2007.1457

Graham, J. W., Cumsille, P. E., \& Elek-Fisk, E. (2003). Methods for handling missing data. In J. A. Schinka \& W. F. Velicer (Eds.) Handbook of psychology, Volume 2: Research Methods in Psychology (pp 87-114). New York: John Wiley \& Sons, Inc. 
Gyselinck, V., Beni, R. D., Pazzaglia, F., Meneghetti, C., \& Mondoloni, A. (2007). Working memory components and imagery instructions in the elaboration of a spatial mental model. Psychological Research, 71(3), 373-382. doi:10.1007/s00426-006-0091-1

Holmes, K. J., \& Lourenco, S. F. (2011). Common spatial organization of number and emotional expression: A mental magnitude line. Brain and Cognition, 77(2), 315-323. doi:10.1016/j.bandc.2011.07.002

Holmes, K. J., \& Lourenco, S. F. (2013). When numbers get heavy: Is the mental number line exclusively numerical? PLoS ONE, 8(3). doi:10.1371/journal.pone.0058381

Hubbard, E. M., Ranzini, M., Piazza, M., \& Dehaene, S. (2009). What information is critical to elicit interference in number-form synaesthesia? Cortex, 45(10), 1200-1216. doi:10.1016/j.cortex.2009.06.011

Jackson, M. C., Morgan, H. M., Shapiro, K. L., Mohr, H., \& Linden, D. E. J. (2011). Strategic resource allocation in the human brain supports cognitive coordination of object and spatial working memory. Human Brain Mapping, 32(8), 1330-1348. doi:10.1002/hbm.21112

Jansari, A. S., Spiller, M. J., \& Redfern, S. (2006). Number synaesthesia: when hearing "four plus five" looks like gold. Cortex, 42(2), 253-258.

Jarick, M., Dixon, M. J., Maxwell, E. C., Nicholls, M. E. R., \& Smilek, D. (2009). The ups and downs (and lefts and rights) of synaesthetic number forms: Validation from spatial cueing and SNARC-type tasks. Cortex 45(10), 1190-1199. doi:10.1016/j.cortex.2009.04.015

Jarick, M., Dixon, M. J., \& Smilek, D. (2011). 9 is Always on top: Assessing the automaticity of synaesthetic number-forms. Brain and Cognition, 77(1), 96-105. doi:10.1016/j.bandc.2011.05.003

Jarick, M., Dixon, M. J., Stewart, M. T., Maxwell, E. C., \& Smilek, D. (2009). A different outlook on time: visual and auditory month names elicit different mental vantage points for a timespace synaesthete. Cortex 45(10), 1217-1228. doi:10.1016/j.cortex.2009.05.014 
Jonas, C. N., Spiller, M. J., Jansari, A., \& Ward, J. (2014). Comparing implicit and synaesthetic number-space associations: Visuospatial and verbal spatial-numerical associations of response codes. The Quarterly Journal of Experimental Psychology, 67(7), 1262-1273. doi:10.1080/17470218.2013.856928

Jonas, C. N., Taylor, A. J. G., Hutton, S., Weiss, P. H., \& Ward, J. (2011). Visuo-spatial representations of the alphabet in synaesthetes and non-synaesthetes. Journal of Neuropsychology, 5(2), 302-322. doi:10.1111/j.1748-6653.2011.02010.x

Mann, H., Korzenko, J., Carriere, J. S. A., \& Dixon, M. J. (2009). Time-space synaesthesia - A cognitive advantage? Consciousness and Cognition, 18(3), 619-627. doi:10.1016/j.concog.2009.06.005

Mohr, H. M., \& Linden, D. E. J. (2005). Separation of the systems for color and spatial manipulation in working memory revealed by a dual-task procedure. Journal of Cognitive Neuroscience, 17(2), 355-366. doi:10.1162/0898929053124929

Mulvenna, C. M., \& Walsh, V. (2006). Synaesthesia: supernormal integration? Trends in Cognitive Sciences, 10(8), 350-352. doi:10.1016/j.tics.2006.06.004

Murray, A. L. (2010). Can the existence of highly accessible concrete representations explain savant skills? Some insights from synaesthesia. Medical hypotheses, 74(6), 1006-1012. doi:10.1016/j.mehy.2010.01.014

Price, M. C. (2009). Spatial forms and mental imagery. Cortex, 45(10), 1229-1245. doi:10.1016/j.cortex.2009.06.013

Price, M. C., \& Mattingley, J. B. (2013). Automaticity in sequence-space synaesthesia: A critical appraisal of the evidence. Cortex, 49(5), 1165-1186. doi:10.1016/j.cortex.2012.10.013

Price, M. C., \& Mentzoni, R. A. (2008). Where is January? The month-SNARC effect in sequence-form synaesthetes. Cortex, 44(7), 890-907. doi:10.1016/j.cortex.2006.10.003 
Rader, C. M., \& Tellegen, A. (1987). An investigation of synesthesia. Journal of Personality and Social Psychology, 52(5), 981-987. doi:10.1037/0022-3514.52.5.981

Rizza, A., \& Price, M. C. (2012). Do sequence-space synaesthetes have better spatial imagery skills? Maybe not. Cognitive Processing, 13(1), 299-303. doi:10.1007/s10339-012-0459-7

Rothen, N., Meier, B., \& Ward, J. (2012). Enhanced memory ability: Insights from synaesthesia. Neuroscience \& Biobehavioral Reviews, 36(8), 1952-1963.

doi:10.1016/j.neubiorev.2012.05.004

Rouw, R., van Driel, J., Knip, K., \& Richard Ridderinkhof, K. (2013). Executive functions in synesthesia. Consciousness and Cognition, 22(1), 184-202.

doi:10.1016/j.concog.2012.11.008

Rusconi, E., Kwan, B., Giordano, B. L., Umiltà, C., \& Butterworth, B. (2006). Spatial representation of pitch height: the SMARC effect. Cognition, 99(2), 113-129. doi:10.1016/j.cognition.2005.01.004

Sagiv, N., \& Robertson, L. C. (2005). Synesthesia and the binding problem. In L. C. Robertson, \& N. Sagiv (Eds.), Synesthesia: Perspectives from cognitive neuroscience. New York: Oxford University Press

Sagiv, N., Simner, J., Collins, J., Butterworth, B., \& Ward, J. (2006). What is the relationship between synaesthesia and visuo-spatial number forms?.Cognition, 101(1), 114-128.

Schafer, J. L., \& Graham, J. W. (2002). Missing data: Our view of the state of the art. Psychological Methods, 7(2), 147-177. doi:10.1037/1082-989X.7.2.147

Seron, X., Pesenti, M., Noël, M. P., Deloche, G., \& Cornet, J. A. (1992). Images of numbers, or “When 98 is upper left and 6 sky blue." Cognition, 44(1-2), 159-196.

Simner, J. (2009). Synaesthetic visuo-spatial forms: viewing sequences in space. Cortex 45(10), 1138-1147. doi:10.1016/j.cortex.2009.07.001 
Simner, J. (2012). Defining synaesthesia. British Journal of Psychology, 103(1), 1-15. doi:10.1348/000712610X528305

Simner, J., Mayo, N., \& Spiller, M.-J. (2009). A foundation for savantism? Visuo-spatial synaesthetes present with cognitive benefits. Cortex, 45(10), 1246-1260. doi:10.1016/j.cortex.2009.07.007

Smilek, D., Callejas, A., Dixon, M. J., \& Merikle, P. M. (2007). Ovals of time: Time-space associations in synaesthesia. Consciousness and Cognition, 16(2), 507-519. doi:10.1016/j.concog.2006.06.013

Spiller, M. J., \& Jansari, A. S. (2008). Mental imagery and synaesthesia: Is synaesthesia from internally-generated stimuli possible? Cognition, 109(1), 143-151. doi:10.1016/j.cognition.2008.08.007

Tang, J., Ward, J., \& Butterworth, B. (2008). Number forms in the brain. Journal of Cognitive Neuroscience, 20(9), 1547-1556.

Terhune, D. B., Rothen, N., \& Cohen Kadosh, R. (2013). Correcting misconceptions about synaesthesia. Neurobiology of learning and memory, 103C, 1-2. doi:10.1016/j.nlm.2013.02.004

Terhune, D. B., Wudarczyk, O. A., Kochuparampil, P., \& Cohen Kadosh, R. (2013). Enhanced dimension-specific visual working memory in grapheme-color synesthesia. Cognition, 129(1), 123-137.

Wagenmakers, E.-J., Maas, H. L. J., Dolan, C. V., \& Grasman, R. P. P. P. (2008). EZ does it! Extensions of the EZ-diffusion model. Psychonomic Bulletin \& Review, 15(6), 1229-1235. doi:10.3758/PBR.15.6.1229

Wagenmakers, E.-J., Maas, H. L. J., \& Grasman, R. P. P. P. (2007). An EZ-diffusion model for response time and accuracy. Psychonomic Bulletin \& Review, 14(1), 3-22. doi:10.3758/BF03194023 
Walsh, V. (2003). A theory of magnitude: common cortical metrics of time, space and quantity. Trends in cognitive sciences, 7(11), 483-488.

Ward, J., \& Sagiv, N. (2007). Synaesthesia for finger counting and dice patterns: a case of higher synaesthesia? Neurocase, 13(2), 86-93. doi:10.1080/13554790701300518

Ward, J., Sagiv, N., \& Butterworth, B. (2009). The impact of visuo-spatial number forms on simple arithmetic. Cortex, 45(10), 1261-1265. doi:10.1016/j.cortex.2009.03.017

Wood, G., Willmes, K., Nuerk, H. C., \& Fischer, M. H. (2008). On the cognitive link between space and number: A meta-analysis of the SNARC effect.Psychology Science Quarterly, 50(4), 489.

Zamarian, L., Ischebeck, A., and Delazer, M. (2009). Neuroscience of learning arithmeticEvidence from brain imaging studies. Neuroscience \& Biobehavioral Reviews 33, 909-925. 


\section{Footnotes}

${ }^{1}$ Twenty of the 23 TNS synaesthetes in this study had both time-forms and numberforms. Two TNS synaesthetes reported only time forms and one reported only number forms. The synaesthetes with time forms did not significantly differ in task performance from the participant with number forms, and the exclusion of these three participants from the main analyses did not change the results. Therefore, the results reported here include all 23 TNS synaesthetes.

${ }^{2}$ It is possible that participants would intentionally or unintentionally estimate the magnitude of time duration before and after the target in order to complete the sequence manipulation of the Time task. To reduce this possibility, it was emphasised to participants they should judge the target's sequential position, and that the duration of time is an unreliable indicator of the number of circles in the sequence.

It is also possible that participants would attempt to estimate the numerosity of stimuli before and after the target in order to complete the sequence manipulation of the Time Task. Even if this were the case, their responses must also reflect a temporal perception of when the target occurred in relation to the rest of the sequence of stimuli.

${ }^{3}$ Box's test and Levene's test were insignificant. Mauchly's test was significant for load $\left(W(5)=.82\right.$, approx. $\left.\chi^{2}=12.34, p=.03\right)$, so the Greenhouse-Geisser values are reported. 
Table 1

Spatial form inducers reported by TNS synaesthetes

\begin{tabular}{lll}
\hline Spatial form inducer & Frequency $(N=23)$ \\
\hline Numbers & & 21 \\
Time: & Hours & 4 \\
& Days & 18 \\
& Weeks & 5 \\
& Months & 20 \\
& Years & 17 \\
Alphabet $^{\mathrm{a}}$ & & 10 \\
Temperature $^{\text {Bank balance }}$ & & 2 \\
\hline
\end{tabular}

${ }^{a}$ We did not specifically recruit synaesthetes with alphabet forms because the alphabet does not represent a magnitude and thus would not allow us to distinguish between magnitude and sequence accounts of TNS synaesthesia, which was one of the aims of this study.

Table 2

Main effects of synaesthesia on Space accuracy with each Working Memory covariate ANCOVA

\begin{tabular}{lcccc} 
Working Memory covariate & $F$ & $d f$ & $p$ & $\eta_{\mathrm{p}}{ }^{2}$ \\
\hline Angle matching accuracy $^{\mathrm{a}}$ & 2.92 & 2,59 & .06 & .09 \\
Colour matching accuracy $^{\mathrm{a}}$ & 2.98 & 2,58 & .06 & .09 \\
Dual matching accuracy $^{\mathrm{b}}$ & 4.42 & 2,59 & .02 & .13
\end{tabular}

${ }^{a}$ The covariate did not have a significant effect on Space accuracy, $p>.05$

${ }^{\mathrm{b}}$ The covariate had a significant effect on Space accuracy, $F(1,59)=8.84, p=.004, \eta_{\mathrm{p}}{ }^{2}=.13$ 


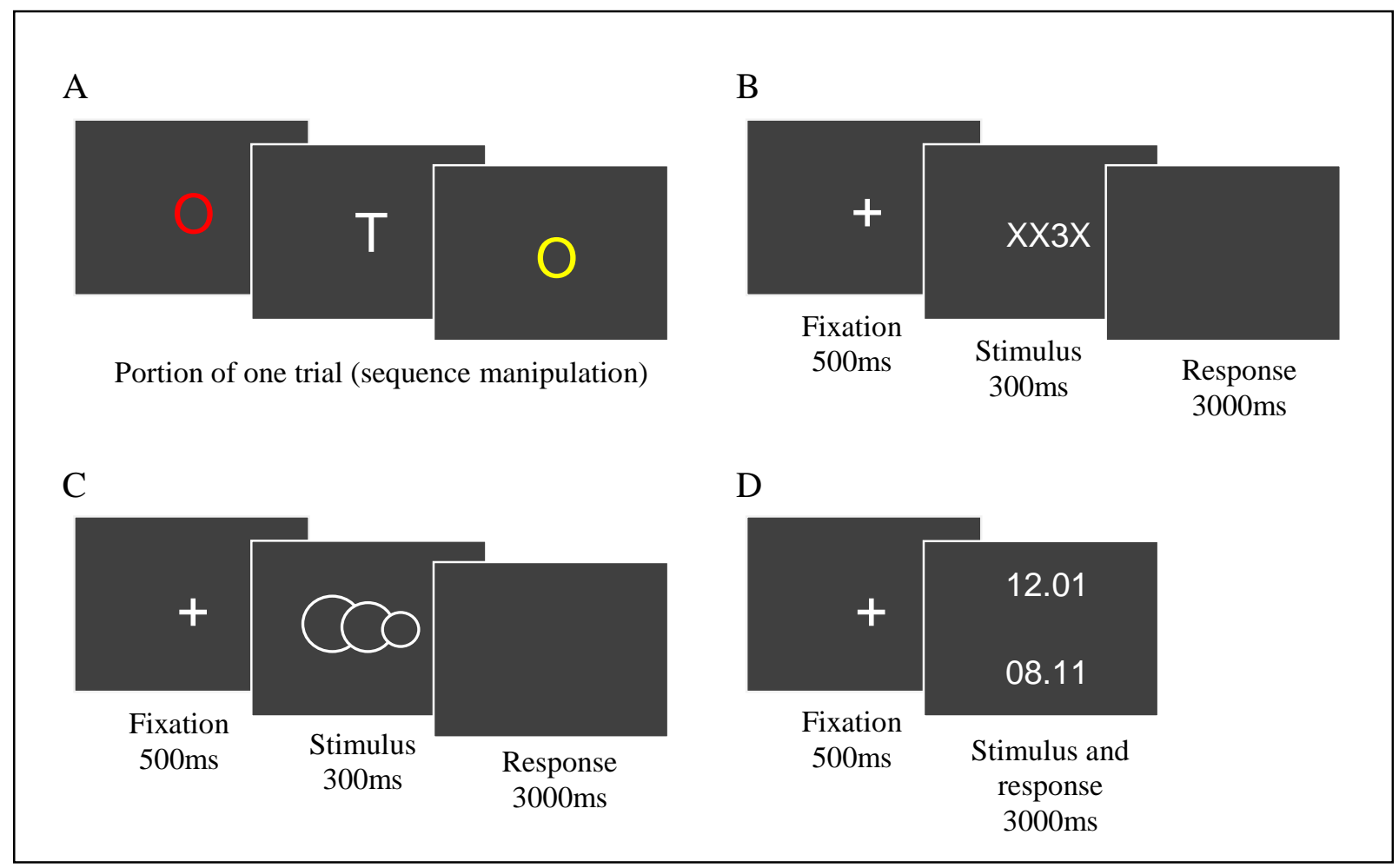

Figure 1. Examples of stimuli used in each trial of the (a) Time, (b) Number, (c) Space and (d) Clock-Date tasks.

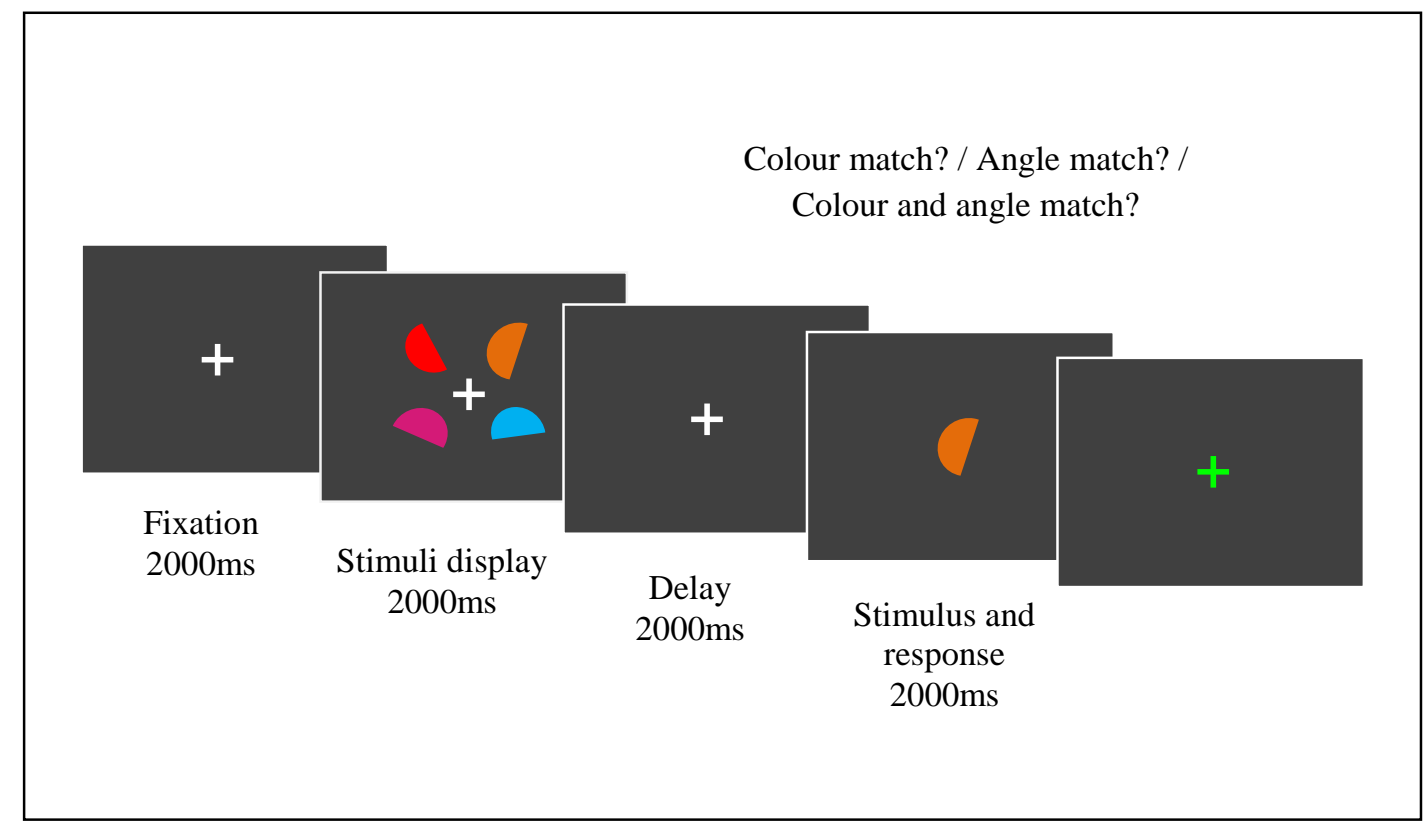

Figure 2. Example stimuli for one trial of the Working Memory task. 


\section{Time}

Magnitude

Sequence

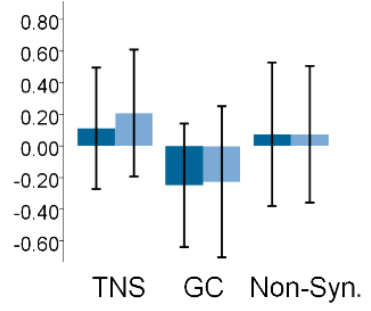

\section{Number}

Magnitude
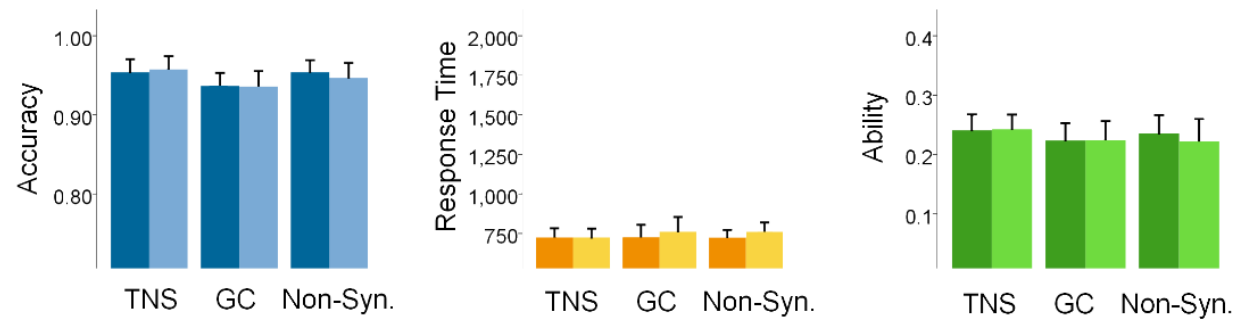

\section{Clock-Date}

Clock
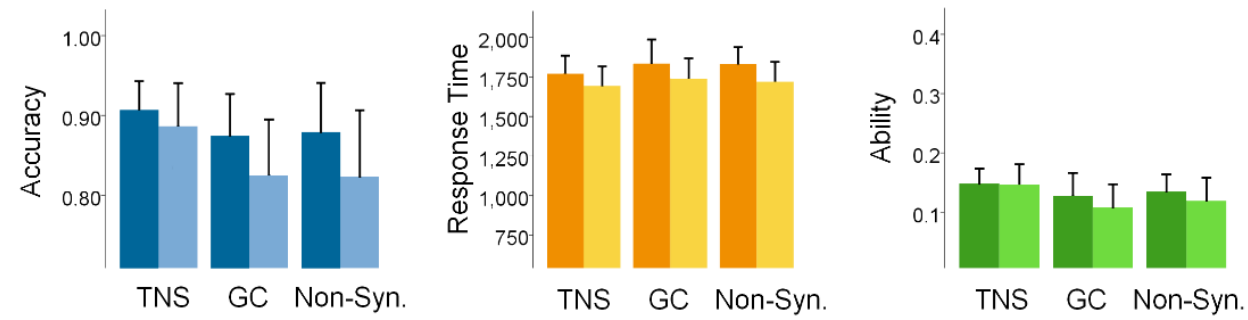

\section{Space}
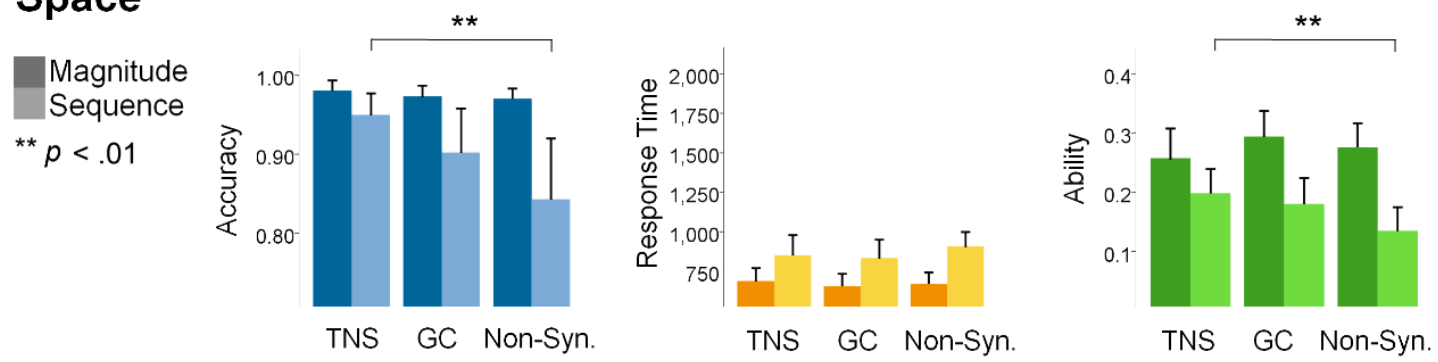

Figure 3. Mean accuracy, response time (milliseconds) and ability of each group on each task manipulation. Error bars represent $95 \%$ confidence intervals. Time task data (note the different scaling) are presented as $z$-scores for comparison between manipulations. 


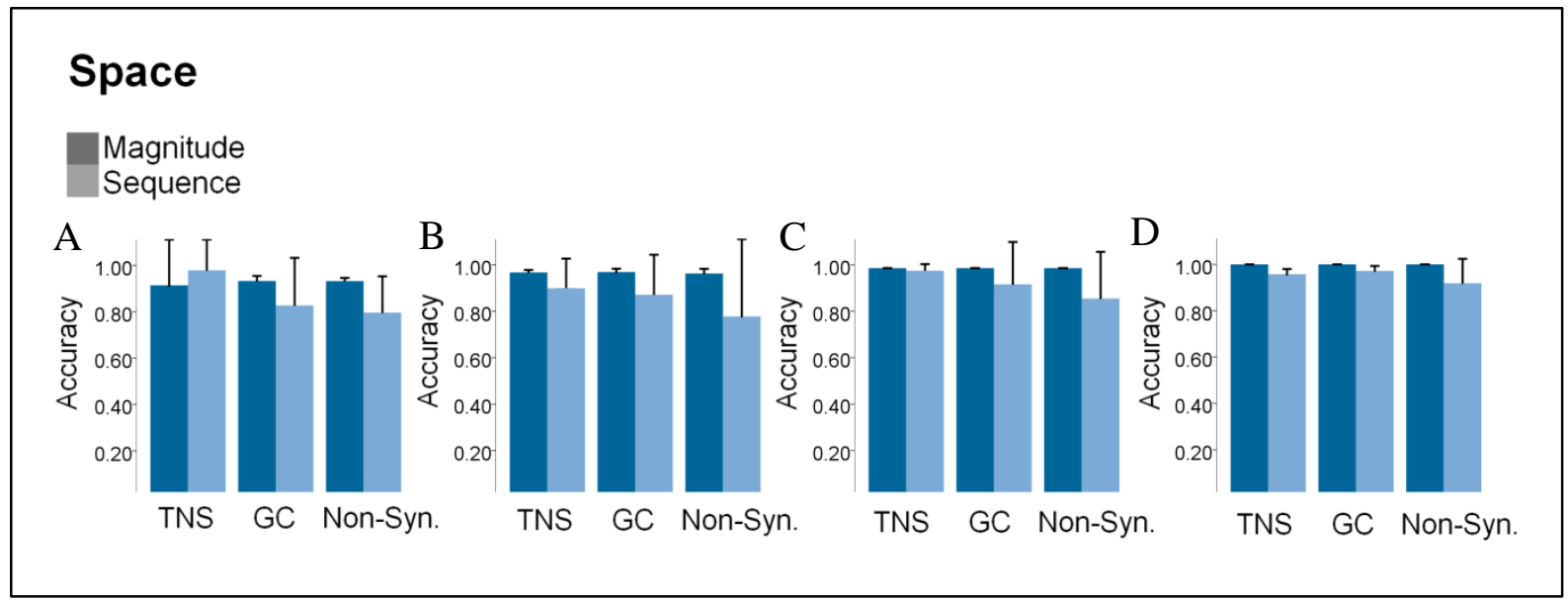

Figure 4.Mean accuracy of each group on each Space task manipulation, by level of magnitude performance, (a) first quartile, (b) second quartile, (c) third quartile, (d) fourth quartile. Error bars represent $95 \%$ confidence intervals. 


\section{Working Memory}

Angle

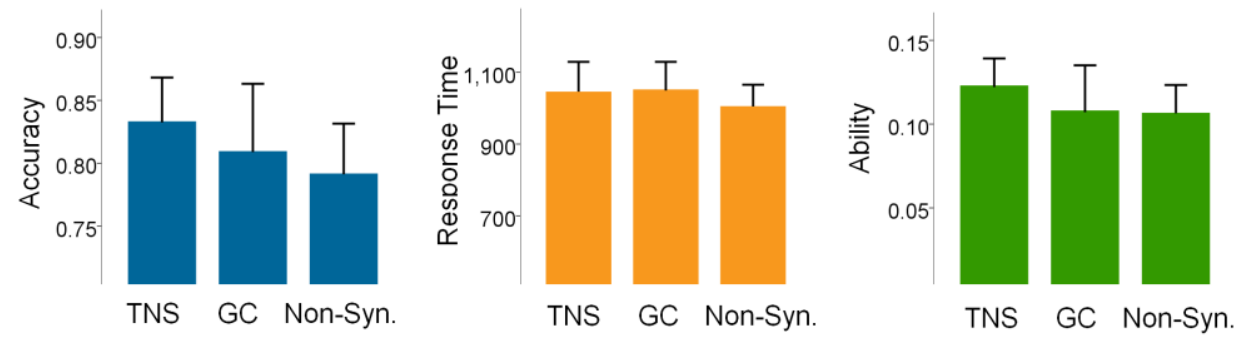

Colour
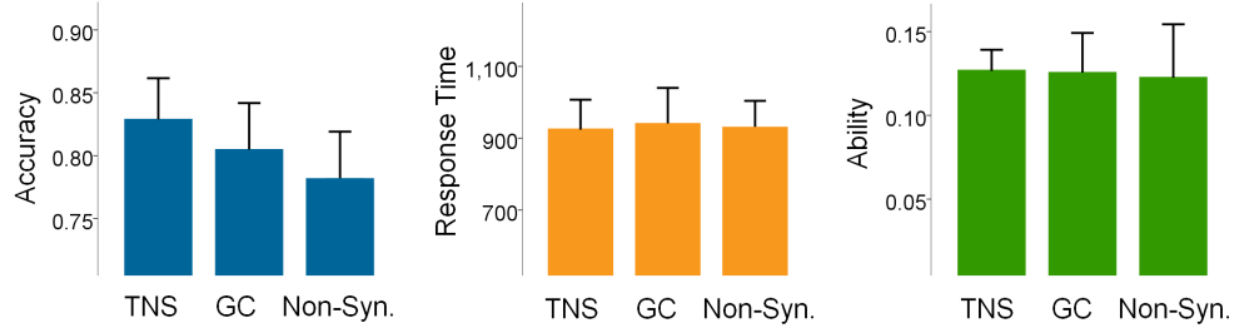

Dual
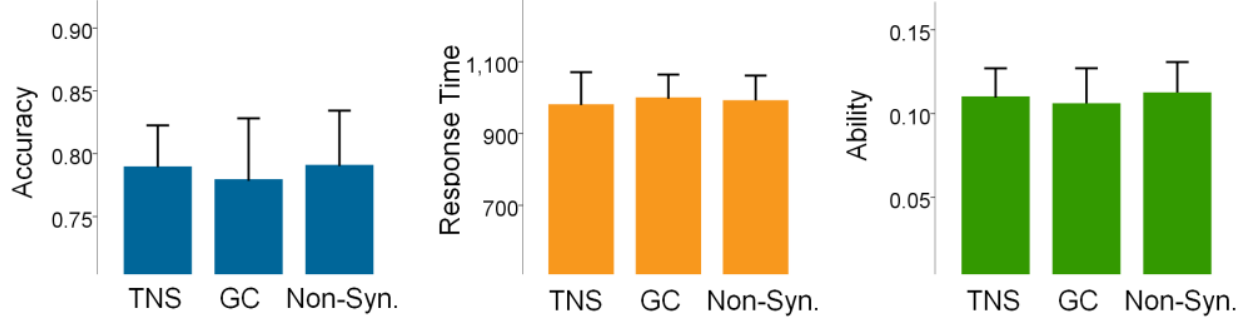

Figure 5. Mean accuracy, response time (milliseconds) and ability of each group on each task manipulation. Error bars represent $95 \%$ confidence intervals. Note the scales in this figure are different from Figures 3-4. 


\section{Appendix}

\section{Task Stimuli}

Time. Coloured circles (white, pink, red, green, yellow, grey, brown and blue) with a diameter of $1.72^{\circ}$ of visual angle were presented one at the time in the centre of the computer screen with mid-grey background of luminance $44 \mathrm{~cd} / \mathrm{m}^{2}$. The stimulus presentation times for each trial were sampled randomly from one of two continuous ranges: fast (200-1000 ms) and slow (1001-2000 ms). Each range was sampled evenly. There were 24 trials in each manipulation of the task. There were four trial durations, 15, 30, 45 and 60s, which were sampled evenly. The order of the trials was randomised for each participant.

Each trial started with a central white fixation cross which remained on the screen until the participant pressed the spacebar. Stimuli were then presented one at a time in the central position (inter-stimulus interval 100ms) until the selected duration was completed. The end of the trial was indicated by the presentation of another central fixation cross, when participants were asked to make a response.

In the sequence manipulation, a white capital letter $\mathrm{T}$ appeared in the centre of the screen for $1000 \mathrm{~ms}$. This time was chosen so as to be consistent with the presentation of stimuli in fast and slow trials. The T appeared at one of eight points during each trial, after $30,35,40,45,55,60,65,70$ or $75 \%$ of the total number of stimuli for that trial had been presented. Each point was sampled evenly.

Number. Strings of characters were presented in the centre of the computer screen. Each string appeared in white Verdana font on a mid-grey background. One character in each string was a number and the remaining characters were the capital letter $\mathrm{X}$. The number varied from 1 to 7 and the length of the string varied from 3 to 5 characters. The number could appear at any position in the string. The characteristics of the stimuli were not sampled evenly; instead, 36 unique stimuli were selected from the possible combinations of 
characteristics so that each number would appear with similar frequency and the position of each number would be equally sampled from the left and right. These were: $1 \mathrm{XX}, \mathrm{XX} 1, \mathrm{X} 2 \mathrm{X}$, 3XX, X3X, XX3, 4XX, XX4, 5XX, XX5, 1XXX, XXX1, X2XX, XX2X, X3XX, XX3X, 4XXX, X4XX, XX4X, XXX4, X5XX, XX6X, 1XXXX, XXXX1, X2XXX, XXX2X, XX3XX, X4XXX, XXX4X, 5XXXX, X5XXX, XX5XX, XXX5X, XXXX5, XXX6X, XXXX7. Each stimulus was presented three times over 108 trials in each block of the task. There were two blocks in each manipulation of the task. The ratio of trails eliciting a correct response of 'yes' vs. 'no' was 1:2.

Each trial started with a central white fixation cross which remained on the screen for $500 \mathrm{~ms}$. Then the stimulus was presented in the central position for 300ms. After each presentation, the screen remained blank until the participant made a response or for up to $3000 \mathrm{~ms}$. The start of the next trial was indicated by the presentation of the central fixation cross.

Space. The Space task stimuli consisted of a set of three overlapping circles, presented in the centre of the computer screen. The circles were mid-grey with a $5 \mathrm{pt}$ white outline and appeared on a mid-grey background. In each set of circles, there was one large, one medium ( $70 \%$ of the large size) and one small ( $70 \%$ of the medium size). The circles overlapped horizontally, in three possible arrangements: the leftmost on top of the middle, on top of the rightmost; the rightmost on top of the middle, on top of the leftmost; or the middle on top of both the leftmost and rightmost. The arrangements were sampled evenly. There were six possible combinations for the positions of the large, medium and small circles, which were also sampled evenly. There were 18 unique stimuli; each was presented twice over 36 trials in each task manipulation. The ratio of trails eliciting a correct response of 'yes' vs. 'no' was 1:2. 
Each trial started with a central white fixation cross which remained on the screen for $500 \mathrm{~ms}$. Then the stimulus was presented in the central position for $300 \mathrm{~ms}$. After each presentation, the screen remained blank until the participant made a response or for up to $3000 \mathrm{~ms}$. The start of the next trial was indicated by the presentation of the central fixation cross.

Clock-Date. The Clock-Date task stimuli consisted of a pair of numbers, presented in the centre of the computer screen, above and below a central white fixation cross. The numbers appeared in white Verdana font on a mid-grey background. Each number had two digits before and after a decimal point. The digits before the decimal point could range from 01 to 12 , and the digits after the decimal point could range from 01 to 28 (to be consistent with the digits which comprise the time on a digital clock or the date in DD.MM format). The position of the larger number (above or below the fixation cross) was sampled evenly. Because there were so many possible combinations of trials, suitable trials (i.e. where the numbers had different digits after the decimal point) were selected randomly. There were 48 unique stimuli, each presented once in each task manipulation. The ratio of trails eliciting a correct response of 'yes' vs. 'no' was 1:2.

These were the pairs of numbers presented:

$\begin{array}{llllll}13.08 & 02.03 & 16.10 & 17.05 & 06.03 & 11.02 \\ 23.10 & 03.02 & 04.07 & 20.05 & 18.10 & 06.11 \\ 05.07 & 09.10 & 04.11 & 20.07 & 23.04 & 13.05 \\ 12.09 & 07.04 & 02.10 & 06.08 & 14.02 & 12.04 \\ 18.06 & 11.03 & 09.05 & 14.02 & 04.12 & 17.08 \\ 15.02 & 18.12 & 03.10 & 11.06 & 08.07 & 23.03 \\ 23.12 & 01.02 & 17.03 & 11.04 & 22.01 & 18.02 \\ 23.06 & 17.02 & 08.04 & 19.02 & 17.10 & 02.12 \\ 13.09 & 20.12 & 01.12 & 18.05 & 17.02 & 08.05 \\ 08.02 & 18.07 & 05.03 & 15.01 & 19.01 & 15.07 \\ 14.06 & 02.04 & 19.08 & 13.12 & & \\ 21.05 & 23.11 & 01.10 & 07.03 & & \\ 14.05 & 18.10 & 15.03 & 21.02 & & \\ 14.03 & 12.01 & 01.11 & 17.07 & & \\ 09.01 & 19.12 & 20.06 & 08.10 & & \\ 01.03 & 05.08 & 07.11 & 16.09 & & \\ 14.08 & 01.11 & 02.11 & 10.09 & & \end{array}$


Each trial started with a central white fixation cross which remained on the screen for $500 \mathrm{~ms}$. Then the stimulus was presented in the central position until the participant made a response or for up to $3000 \mathrm{~ms}$. The start of the next trial was indicated by the presentation of the central fixation cross (inter-trial interval 100ms). 